УДК 53.097

DOI: $10.21779 / 2542-0321-2016-31-4-36-40$

\title{
Д.С. Лапицкий
}

\section{Сепарация микрочастиц с помощью линейной ловушки Пауля}

Федеральное государственное бюджетное учреждение науки Объединенный институт высоких температур РАН (ОИВТ РАН); Россия, 125412, Москва, ул. Ижорская, 13, cmp. 2; dmitrucho@yandex.ru

В работе продемонстрирована возможность сепарации частиц с помощью переменных электрических полей квадрупольного типа, создаваемых с помощью линейных ловушек Пауля. Было показано, что изменяя параметры ловушки (расстояния между электродами, величину и частоту переменного напряжения, подаваемого на электроды ловушки), возможно как отделять от порошка частицы определенного размера, так и пространственно разделять частицы внутри ловушки.

Ключевые слова: сепаращия частии, ловушка Пауля, монодисперсные порошки.

\section{Введение}

Монодисперсные порошки из микронных и субмикронных частиц с известными параметрами часто используются в экспериментах в физике низкотемпературной плазмы [1-4], в PIV методах исследований газовых потоков [5, 6], широко распространены в области нанотехнологий [7]. Таким образом, создание монодисперсных порошков является актуальной задачей.

Существует несколько возможностей для получения монодисперсных порошков. В работе [8] сепарация частиц достигается путем их самоорганизации в узлах поверхностных стоячих волн. Частицы различного размера собираются в определенные области из-за пространственного распределения силы акустического давления, чья величина различна в различных областях устройства.

В [9] сепарация частиц достигается за счет диэлектрофореза. Наиболее распространённым является способ сепарации за счет циклонов, с помощью которых можно разделять как жидкие и твердые фазы из газов [10], так и частицы с различными массами. Сепарация частиц в высокочастотном и тлеющем разрядах представлена в [11].

В масс-спектрометрии сепарация ионов часто производится в ловушках Пауля [12]. Движение ионов в вакууме в ловушках Пауля описывается системой уравнений Матье. В воздухе при атмосферном давлении за счет диссипации энергии область параметров, при которых происходит удержание частицы, становится шире [13]. В работах [14-15] изучался процесс захвата и удержания микрочастиц переменными электрическими полями, формируемыми ловушками Пауля, в неподвижных газовых средах и в газовых потоках.

Цель данной работы - теоретические и экспериментальные исследования возможности сепарации заряженных микрочастиц из полидисперсных порошков с помощью переменных электрических полей, формируемых линейными ловушками Пауля. 


\section{Модель расчета динамики микрочастиц}

В данной работе исследовались области удержания заряженной микрочастицы в линейной ловушке Пауля [13] как зависимости от размера и заряда частицы, а также частоты переменного напряжения, подаваемого на электроды ловушки.

Для описания поведения микрочастицы в переменных электрических полях в воздухе при атмосферном давлении использовался метод броуновской динамики, учитывающий стохастические силы, действующие на микрочастицу благодаря её столкновениям с нейтральными частицами буферного газа, силы взаимодействия частицы с переменными полями ловушки, силу тяжести. Динамика частицы в таком случае описывается уравнением Ланжевена [22]:

$$
m_{p} \frac{d^{2} r}{d t^{2}}=F_{t r}(r)-6 \pi \eta r_{p} \frac{d r}{d t}+F_{b}+F_{m g},
$$

где $m_{\mathrm{p}}$ и $r_{\mathrm{p}}$ - это масса и радиус микрочастицы; $\eta$ - динамическая вязкость среды; $F_{\mathrm{tr}}(r)$ - сила взаимодействия электрического поля ловушки с заряженной микрочастицей; $F_{\mathrm{b}}$ - сила, описывающая столкновения микрочастицы с частицами буферного газа; $F_{m g}-$ вес микрочастицы. Для расчета силы взаимодействия электрического поля ловушки с заряженной частицей использовалась модель точечных зарядов, равномерно распределенных вдоль электродов ловушки [13].

В предположении, что частицы приобретают заряд в коронном разряде, на рисунке 1 представлены области удержания микрочастицы как зависимость частоты переменного напряжения $f$ от объемного заряда частицы $\sigma_{\mathrm{p}}=q_{\mathrm{p}} / 4 \pi r_{\mathrm{p}}{ }^{2}$, размера частицы и межэлектродного расстояния ловушки. Рисунок $1 a$ соответствует ловушке с межэлектродным расстоянием $1,3 \mathrm{~cm}$, а $16-2,8 \mathrm{~cm}$. Как видно из рисунка 1 , чем меньше межэлектродное расстояние, тем меньше может быть заряд частицы для ее удержания, кроме того, области удержания частицы зависят от ее размера.

В коронном разряде частица приобретает заряд, который зависит от напряженности электрического поля разряда $E_{\mathrm{c}}$, а также площади поверхности частицы $q_{\mathrm{m}}=$ $\pi r_{\mathrm{p}}{ }^{2} \varepsilon_{0} E_{\mathrm{c}}(1+2(\varepsilon-1) /(\varepsilon+2))[25]$, где $\varepsilon-$ диэлектрическая проницаемость материала частицы (в расчетах $\varepsilon=10)$. На рисунке 1 правые границы соответствуют максимальной плотности заряда, которую может приобрести частица в коронном разряде с напряженностью электрического поля $E_{\mathrm{c}}$, т. е. эти границы могут сдвигаться как влево, так и вправо, за счет чего появляется возможность настроить работу ловушки на удержание определенного размера частицы.

Так, для маленькой ловушки на рисунке $1 a$ частицы с радиусами 10 мкм возможно удержать при наименьшей величине плотности заряда по сравнению с частицами других размеров. А «отсечь» частицы других размеров можно сдвинув границу, т. е. уменьшить $E_{\mathrm{c}}$, как, например, на рисунке 16 вертикальной границей отсечены частицы c $r_{\mathrm{p}}=20$ мкм. Помимо этого, изменяя частоту, можно добиться удержания определенных сортов частиц. Так, на рисунке $1 a$ частицы с $r_{\mathrm{p}}=20$ мкм невозможно удержать при частотах меньше 50 Гц, а на рисунке 16 при низких частотах невозможно удержать как 20 мкм частицы, так и 10 мкм. 

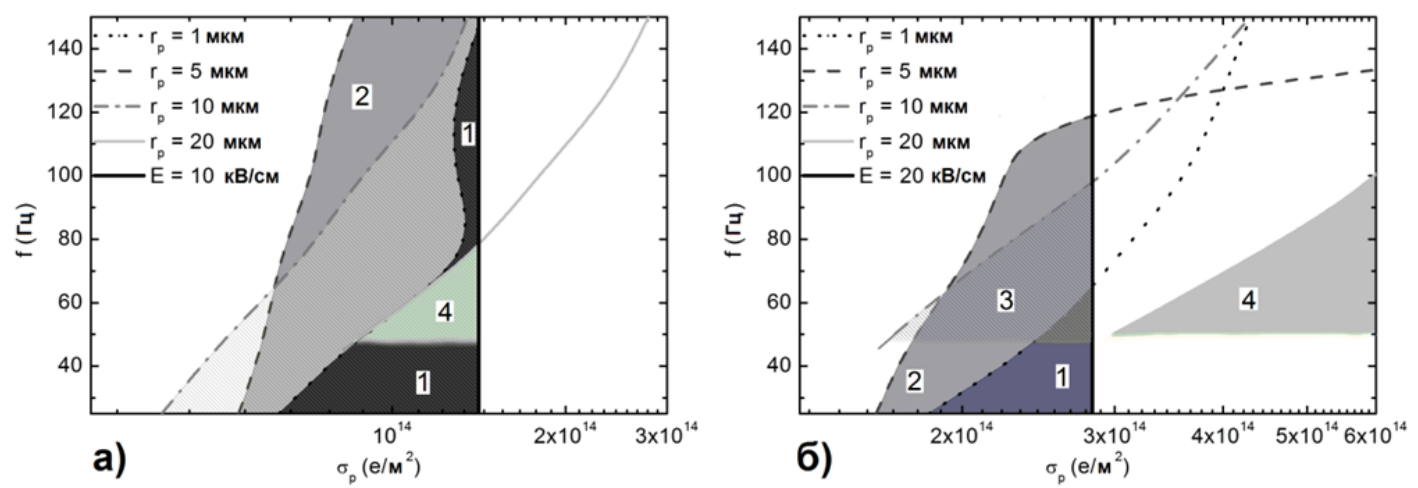

Рис. 1. Области захвата микрочастицы как зависимость частоты $\mathrm{f}$ переменного напряжения от плотности заряда частицы для различных размеров частиц и геометрий ловушек: а) межэлектродное расстояние 1,3 см, б) межэлектродное расстояние 2,8 см. Амплитуда переменного напряжения 5 кB

\section{Эксперимент по сепарации заряженных частиц в ловушке Пауля}

Схема экспериментальной установки представлена на рисунке $2 a$. Установка состояла из двух основных модулей, расположенных в газовом тракте. Модуль для формирования коронного разряда 1 , предназначенный для зарядки частиц, и модуль ловушки Пауля 2 [17-дгу], где происходил процесс захвата заряженных частиц и их сепарация. Ловушка Пауля состояла из четырех цилиндрических электродов (1 и 2 на рисунке 1) радиусами $R_{1}=1.5$ мм и длинами $L_{\mathrm{m}}=30 \mathrm{~cm}$. На электроды ловушки подавалось переменное напряжение $U_{\omega} \sin (\omega t)$ и $U_{\omega} \sin (\omega t+\pi), U_{\omega}=10$ кB, $f=2 \pi \omega=50$ Гц. Для исследования влияния размеров ловушки на сепарацию частиц два верхних электрода были наклонены таким образом, что у одного основания ловушки торцы электродов располагались в углах квадрата со стороной 1,6 см, а у другого основания торцы электродов располагались по углам прямоугольника длиной 5,5 см и шириной 1,6 см.

Полидисперсный порошок частиц оксида алюминия инжектировался в модуль коронного разряда, где частицы приобретали заряд, и после этого частицы осаждались под действием силы тяжести уже в ловушку, где происходил их захват и расслоение на два четко выраженных облака, как показано на рисунке 26.

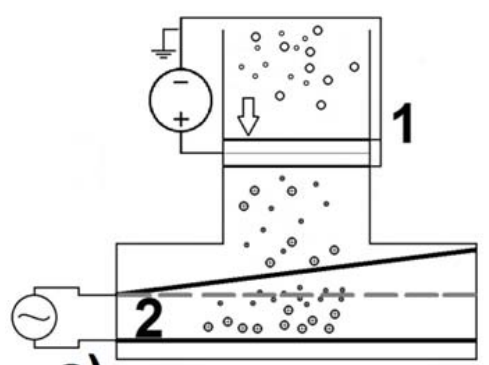

a)

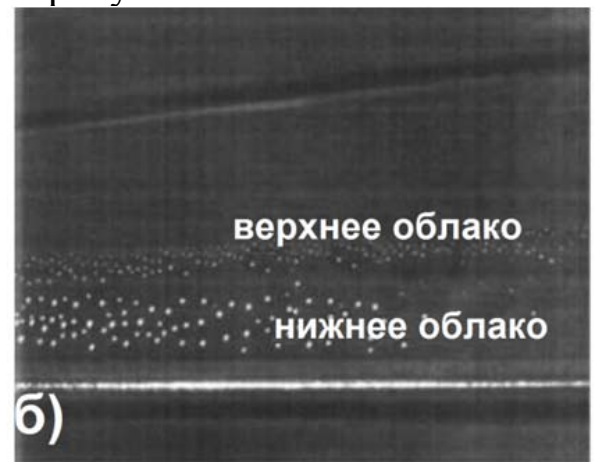

Рис. 2: а) схема установки для сепарации частиц, б) пространственная сепарация частиц с помощью переменных электрических полей

Благодаря свободному пространству между облаками было возможно собрать частицы независимо на предметное стекло. Чтобы частицы увлекались на стекло, за стеклом был установлен электрод, на который подавался постоянный потенциал, противоположный по знаку заряду частиц. Размеры частиц исследовались с помощью микроскопа. 
Распределения частиц по размерам представлены на рисунке 3. Диаметр частицы рассчитывался как среднее арифметическое от наибольшего и наименьшего размера частицы. На рисунке 3 линия 1 соответствует распределению частиц, прошедших модуль коронного разряда, 2 - захваченных в верхнем облаке, 3 - в нижнем. Средний размер частиц для верхнего облака составил 2,85 мкм, для нижнего - 5,5 мкм.

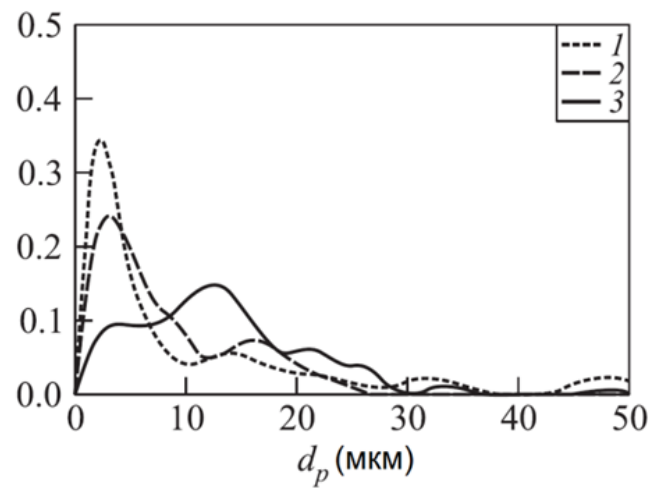

Рис. 3. Распределение частиц по размерам. 1- исходное распределение частиц после их прохождения модуля коронного разряда, 2 - верхнее облако, 3 - нижнее облако

\section{Вывод}

В работе теоретически показана возможность сепарации частиц с помощью переменных электрических полей. Показано, что изменяя параметры установки (влияющие в том числе на процесс зарядки частиц), возможно как настроить установку на захват частиц определенного сорта, так и пространственно разделять частицы.

Работа была выполнена при финансовой поддержке гранта РФФИ 16-32-00031 мол-а.

\section{Литература}

1. Vishnyakov V.I. and Dragan G.S. Coupling parameter for low-temperature plasma with condensed phase // Condensed Matter Physics. - 2007. - V. 10, № 2(50). - P. 201-208.

2. Vishnyakov V.I. and Dragan G.S. Thermodynamic reasons of agglomeration of dust particles in the thermal dusty plasma // Condensed Matter Physics, 2003, V.6, N.4 (36), P.687-692

3. Smith B., Hyde T., Matthews L., Reay J., Cook M. and Schmoke J. Phase transitions in a dusty plasma with two distinct particle sizes // Advances in Space Research. - 2008 V. 41 (9). - P. 1510-1513.

4. Фортов В.Е., Храпак А.Г., Храпак С.А., Молотков В.И., Петров О.Ф. Пылевая плазма // УФН - 2004. - Т. 174. - С. 495-544.

5. Melling $A$. Tracer particles and seeding for particle image velocimetry // Meas. Sci. Technol. - 1997. - V. 8. - P. 1406-1416.

6. Glushniova A.V., Saveliev A.S., Son E.E., Tereshonok D.V. Shock wave-boundary layer interaction on the non-adiabatic ramp surface // High Temperature. - 2014. - V. 52 (2). - P. 220-224.

7. Privman $V$. Growth of Nanosize and Colloid Particles by Controlled Addition of Singlets // Mat. Res. Soc. Symp. Proc. - 2002. - V. 703, Article T3.3. - P. 577-585.

8. Rasim Guldiken, Myeong Chan Jo, Nathan D. Gallant, Utkan Demirci, Jiang Zhe. Sheathless Size-Based Acoustic Particle Separation // Sensors. - 2012. - V. 12 (1). - P. 905922. 
9. Gascoyne P.R., Vykoukal J. Particle separation by dielectrophoresis // Electrophoresis. $-2002 .-$ V. 23 (13). - P. 1973-83.

10. Bert Brouwers. Rotational particle separator: A new method for separating |ne particles and mists from gases // Chem. Eng. Technol. - 1996. - V. 19. - P. 1-10.

11. Batryshev D.G., Ramazanov T.S., Dosbolayev M.K., Gabdullin M.T. Separation Process of Polydisperse Particles in the Plasma of Radio-frequency Discharge // J. NanoElectron. Phys. - 2014. - V. 6, № 3. - P. 03032.

12. Mihalcea B.M., Visan G.T., Giurgiu L.C., Radan S. Optimization of ion trap geometries and of the signal-to-noise ratio for high resolution spectroscopy // J. Optoelectron. Adv. Mater. - 2008. - V. 10 (8). - P. 1994-1998.

13. Lapitsky D.S., Filinov V.S., Deputatova L.V., Vasilyak L.M., Vladimirov V.I., Pecherkin V.Ya. Capture and retention of charged dust particles in electrodynamic traps // High Temp. - 2015. - V. 53 (1). - P. 1-8.

14. Vasilyak L.M., Vladimirov V.I., Deputatova L.V., Lapitsky D.S., Molotkov V.I., Pecherkin V.Ya., Filinov V.S., Fortov V.E. Coulomb stable structures of charged dust particles in a dynamical trap at atmospheric pressure in air // New J. Phys. - 2013. - V. 15. P. 043047.

15. Lapitsky D.S., Filinov V.S., Deputatova L.V., Vasilyak L.M., Vladimirov V.I., Pecherkin V.Ya. Confinement of the charged microparticles by alternating electric fields in a gas flow // Europhys. Lett. - 2015. - V. 110. - P. 15001.

Поступила в редакичию 29 октября 2016 г.

UDC 53.097

DOI: $10.21779 / 2542-0321-2016-31-4-36-40$

\section{Separation of microparticles in linear Paul trap}

\section{D.S. Lapitsky}

Joint Institute for High Temperatures RAS; Russia, 125412, Moscow, Izhorskaya st., 13; bd.2;dmitrucho@yandex.ru

The paper demonstrates the possibility of particle separation by means of alternating electric fields of the quadrupole type, generated by the linear Paul trap. It was shown that by changing the parameters of the trap (the distance between the electrodes, the magnitude and frequency of the AC voltage applied to the trap electrodes) it is possible to separate particles of a certain size from polydisperse powder of particles as well as spatially separate particles in the trap.

Keywords: particle separation, Paul trap, monodisperse powders.

Received 29 October, 2016 